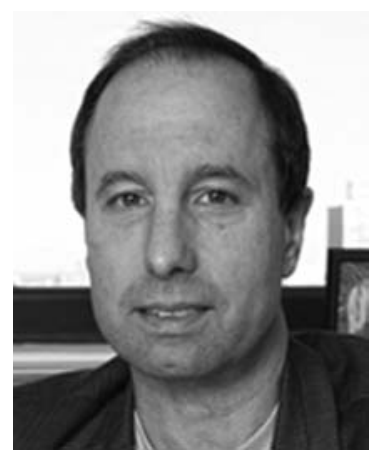

\title{
A Conversation with Michael Shadlen
}

\author{
INTERVIEWER: ANN GOLDSTEIN \\ Scientific Editor, Neuron
}

Michael Shadlen is a Professor in the Department of Neuroscience, The Kavli Institute for Brain Science at Columbia University and a Howard Hughes Medical Institute Investigator.

Ann Goldstein: What led you to start asking the questions about how decisions are made and decoded in the brain?

Dr. Shadlen: I did my postdoctoral training with Bill Newsome who was the main pioneer in this field, and we had worked out with our colleagues the degree to which you could understand the perceptual limits of monkeys and humans, by relating signal-to-noise relationships in the visual cortex to what you could call noise relationships in behavior-that is, errors and accuracy. We did a lot of computational modeling to reconcile neural recordings and behavior and developed a hypothesis about what downstream structures in the brain were doing to render a choice from the data.

I have a background in neurology. I think the neurobiology of decision-making will ultimately provide insights into neurological disorders affecting higher cognitive function - schizophrenia and autism, for example-because decision-making provides a window on the basic building blocks of cognition. Decisions involve some kind of contingency, using information in a flexible timescale. These are the basic elements of thought in general, and the underlying mechanisms - when they go awryare probably modes of failure in disease.

The study of decision-making in normal brains is the study of what makes a normal person not confused. That's what led us to study structures in the brain that might be making decisions about something simple like visual stimuli, but we see this in a broader context such as what constitutes a thought or coming to a belief.

Ann Goldstein: How do you study decision-making in a human subject? How do you build a task to test it?

Dr. Shadlen: Every perceptual experiment in psychology has a decision component, because in the end the subject-human, monkey, or rat - is going to look at something, decide what is being seen, and push a button or make an eye movement. I think we must jettison, or at least be skeptical about, the idea that what we know is what we represent in the brain. Instead what we know about the world may come from how the information we receive bears on the hypothesis we have about the world. If I make an answer with a button box, I've got to figure out what it is in my brain that made me move my thumb to push the button. I want to define a decision process backwards, starting with the behavior.

We ask "What's happening in the brain?" and replaced this with a more refined question: "Since I will answer, ultimately, with an eye movement, or a push of a button, what led the brain to form a plan to push this or that button, gaze toward this or that spot, say the word 'right' or 'left'?" So that led us to put our electrodes into a part of the brain that you wouldn't have expected. We worked backwards from, in our case, the ocular motor system, because monkeys reported their answers about decisions by eye movements, and that led us to the deliberation process - all the steps that led to the decision.

Ann Goldstein: There are going to be new studies using microstimulation in humans, following similar investigations in mice. Is this a powerful tool that will help us understand complex cognitive functions such as decision?

Dr. Shadlen: I think it's extremely strong. Most of what we do in systems neuroscience is correlative and I'm a big defender of correlation. Quantitative connections are valuable, but in the end if you're studying neurons, you'd like to say, "Is this circuit involved and can I manipulate it someway?" There are risks to that. There's no guarantee that the neurons you're activating have direct access to behavior. But causal manipulations are really important, and we're seeing new techniques for causal experiments such as optogenetics. What has worked for a long time is electrical microstimulation, especially in the visual cortex.

Ann Goldstein: You are interested in confidence and its neural mechanisms?

Dr. Shadlen: When we make a decision, there are three parts to it: choice, which we can grade as accurate- 
correct or incorrect; how long it takes - we can compare with accuracy; and a third more elusive component, which is confidence. You can think of confidence as an internal prediction about whether the decision we've just committed to is correct or not.

So how do you get that? Where does it come from? How do you measure it? A colleague in my lab figured out a way to do this by stealing a trick from the cognitive psychologists, where you get monkeys to bet. You say, “Okay, you've made your decision, but you could opt out of it and get a small reward or stick with it and get a big reward if you're right and nothing if you're wrong." That turns out to be a way to measure confidence.

Confidence in the end is some kind of internal implicit knowledge in the brain of the probability that the decision about to be rendered is correct. The mechanisms that explain how signal-to-noise information in the visual system leads to being correct or incorrect, or faster or slower, also explain how you can predict from your own choice whether you're likely to be correct or not.

In recent experiments, we have stimulated the brain and caused changes in choice, the original experiment that Bill Newsome did 20 years ago. But we could show that there were quantitatively commensurate changes caused in the degree of confidence. That is a bit of a shocker, because if you stimulate the brain artificially through these causal manipulations, we have no idea what it really does to a brain, and we give an animal an opportunity to say, "Wait a second, that was whacky, never experienced anything like that before." But they don't tell us that. They say, "That was like vision."

Ann Goldstein: What are you most excited about in this area? What's the next level for understanding decision and how it relates to other higher cognitive processes?

Dr. Shadlen: What we're doing is exposing what I hope will be principles of cognitive neuroscience. I've just eluded to several of them: integration of evidence, having a reason to stop and therefore trade off speed versus accuracy; doing computations in the language of probability theory; belief that I'm going to be correct or not. I think all of those kinds of elements come into play when we're having a dialogue using language and logic and so forth. What I'd like to think is that as we evolved bigger brains and more of the association cortex that supports these simple kinds of decisions, these operations and more to be discovered, came into play. As I mentioned earlier, I believe that these functions are failure modes in diseases that effect higher brain function such as schizophrenia, autism, and confusion states in general. My dream is that we'll be able to treat diseased brains one day by restoring these kinds of functions - integration of evidence, setting bounds, making decisions, and so on. That's a 20-year horizon, at least! We're nowhere near it yet, but you can almost see the path there. 


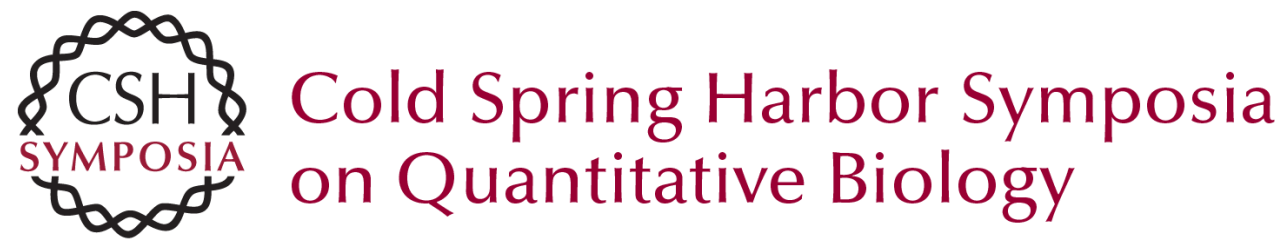

\section{A Conversation with Michael Shadlen}

Cold Spring Harb Symp Quant Biol 2014 79: 291-292

Access the most recent version at doi:10.1101/sqb.2014.79.16

\section{License}

Email Alerting Receive free email alerts when new articles cite this article - sign up in Service the box at the top right corner of the article or click here. 